Conclusions In a high volume Comprehensive Stroke Center, general anesthesia is safe to always use mechanical thrombectomy. Outcomes of mechanical thrombectomy for LVO in are in keeping with published results for both conscious sedation and general anesthesia. This adds to the body of evidence supporting GA as a excellent option for sedation for mechanical thrombectomy. It is particularly useful in more complex cases and leads to high revascularization rates.

Disclosures A. Persad: None. S. Ahmed: None. Z. Tymchak: None. R. Whelan: None. A. Gardner: None. G. Hunter: None. B. Graham: None. L. Peeling: 2; C; Medtronic. M. Kelly: 2; C; Medtronic.

\section{E-146 QUANTITATIVE EVALUATION OF FACTORS AFFECTING TUMOR SIZE REDUCTION ON WIDE ANGLE FUNDUS CAMERA FOLLOWING RETINOBLASTOMA CHEMOSURGERY}

${ }^{1} \mathrm{~S}$ Chen ${ }^{*},{ }^{2} \mathrm{P}$ Chévez-Barrios, ${ }^{3} \mathrm{M}$ Chintagumpala, ${ }^{3} \mathrm{~F}$ Lin, ${ }^{4} \mathrm{P}$ Kan, ${ }^{5} \mathrm{C}$ Area, ${ }^{6} \mathrm{C}$ Herzog, ${ }^{7} \mathrm{D}$ Gombos. ${ }^{1}$ Radiology and Neurosurgery, Baylor College of Medicine/Retinoblastoma Center of Houston, Houston, TX; ${ }^{2}$ Pathology, Retinoblastoma Center of Houston/Houston Methodist Hospital, Houston, TX; ${ }^{3}$ Pediatrics, Hematology-Oncology, Texas Children's Hospital/Retinoblastoma Center of Houston, Houston, TX; ${ }^{4}$ Neurosurgery, Baylor College of Medicine/Retinoblastoma Center of Houston, Houston, TX; ${ }^{5}$ Radiology, Baylor College of Medicine/Retinoblastoma Center of Houston, Houston, TX; ${ }^{6}$ Pediatrics, HematologyOncology, The University of Texas MD Anderson Cancer Center/Retinoblastoma Center of Houston, Houston, TX; ${ }^{7}$ Head and Neck Surgery, The University of Texas MD Anderson Cancer Center/Retinoblastomal Center of Houston, Houston, TX

\subsection{6/neurintsurg-2019-SNIS.221}

Introduction Intra-arterial delivery of chemotherapy (chemosurgery) via selective ophthalmic artery infusion has had an increasing role in the treatment of retinoblastoma. However reported success rates and enucleation rates have varied. The many differences in institutional protocols including choice of first line therapy, availability of patient follow-up and rationale for enucleation yields different results. We hypothesized that a quantitative assessment of tumor size reduction on wide angle funduscopic imaging would show differences in tumor response with regards to cycle of treatment, adjunct treatments, and prior IV chemotherapy.

Materials and methods Following IRB approval, a retrospective review of pediatric patients with retinoblastoma who had been referred for chemosurgery at our institution in the past 6 years was performed. Data collected included patient demographics, retinoblastoma classification, prior and concurrent treatments, chemotherapy agents, catheterization technique, fluoroscopy time, vascular anatomy, complications, and response on funduscopic examination. RetCam wide angle fundoscopic images were digitally transferred to DICOM image processing software for analysis or digitized from hard copy. Quantitative changes in tumor volume between images were measured.

Results From June 2013 to March 2019, a total of 114 chemosurgery cases were performed on 30

patients to treat 32 eyes. All patients received eye exams under anesthesia. 158 eye exams under anesthesia were reviewed including analysis of wide angle fundoscopic images when available.

After the first cycle of intra-arterial chemotherapy, response to treatment progressively decreased, with greatest change in tumor volume at time of first treatment. Decrease tumor size decrease per treatment was much greater when intra-arterial therapy was utilized as a first line therapy.

\begin{tabular}{llll} 
Abstract E-146 Table 1 & & & \\
\hline & Cycle & Cycle 2 & Cycle \\
& 1 & & 3 \\
\hline First Line & $58.9 \%$ & $35.54 \%$ & $2.05 \%$ \\
Prior IV & $4.12 \%$ & $3.97 \%$ & $2.88 \%$ \\
Chemotherapy & & & \\
\hline
\end{tabular}

Combination therapy appears to be more efficacious than IA alone for cycles 3 and greater.

\begin{tabular}{lc}
\hline IA Alone & $1.58 \%$ \\
\hline Concurrent Laser Hyperthermia & $2.89 \%$ \\
Concurrent Intravitreal & $9.30 \%$ \\
Chemotherapy & \\
\hline
\end{tabular}

The catheters were placed in two locations, either the middle meningeal artery or the origin of the ophthalmic artery. A slightly greater average decrease in tumor size per cycle was seen with middle meningeal infusion (17.63\%) versus ophthalmic artery infusion (12.38\%).

Conclusion Quantitative assessment of wide angle fundoscopic imaging for tumor size helps evaluate Chemosurgery efficacy retrospectively. Utilizing this measurement allows us to more accurately assess treatment effects, which appear to be greater in IV chemotherapy naïve patients.

Disclosures S. Chen: None. P. Chévez-Barrios: None. M. Chintagumpala: None. F. Lin: None. P. Kan: 1; C; Stryker Neurovascular, MacDonald Research Fund. 2; C; Stryker Neurovascular, Medtronic. C. Area: None. C. Herzog: None. D. Gombos: 1; C; Houseman/Wilkin Opthalmologicl Foundation. 2; C; Abbvie, Aura, 3T Ophthalmics. 6; C; Children's Oncology Group.

\section{E-147 A RETROSPECTIVE REVIEW OF THE IMPACT OF ASPIRIN USE IN SUBARACHNOID HEMORRHAGE}

${ }^{1}$ V Patel ${ }^{*},{ }^{1} \mathrm{~F}$ Al Mufti, ${ }^{2} \mathrm{R}$ Gupta. ${ }^{1}$ Neurosurgery, Westchester Medical Center, Valhalla, NY; ${ }^{2}$ Neurosurgery, Rutgers New Jersey Medical School, Newark, NJ

\subsection{6/neurintsurg-2019-SNIS.222}

Objective There is limited evidence on the use of aspirin (ASA) to reduce the risk and morbidity of cerebral aneurysmal rupture. This analysis retrospectively assessed aspirin use in patients presenting to our institution with aneurysm rupture.

Methods We evaluated 7 years of retrospective data from our neurovascular center and an existing database of patients with aneurysmal rupture. Initial presentation, hospital course and discharge outcomes were evaluated and compared based on whether or not patients were on ASA at the time of aneurysm rupture.

Results A total of 18 cases with patients on ASA and 168 control patients were identified. ASA use did not appear to lead to differences in initial presentation, including Hunt-Hess score and Fisher grade. In addition, ASA use was not associated with increased rates of delayed cerebral ischemia (DCI) or worsened modified Rankin score (mRs) at 90 days. Our analysis showed that increased Hunt Hess score and the 
presence of DCI are both associated with increased mRS at 90 days.

Conclusions Use of ASA does not appear to confer worse outcomes in cerebral aneurysm rupture and subarachnoid hemorrhage.

Disclosures V. Patel: None. F. Al Mufti: None. R. Gupta: None.

\section{E-148 DECOMPRESSIVE CRANIECTOMY IN ACUTE STROKE PATIENTS IN KOREAN SINGLE CENTER}

K Jang*, B Cho, B Moon, K Kim, D Jang. Neurosurgery, Incheon St. Mary's hospital, The Catholic University of Korea, Bupyeong-gu, Incheon, Korea, republic of

\subsection{6/neurintsurg-2019-SNIS.223}

Introduction Decompressive craniectomy (DC) is a life-saving surgical procedure being increasingly employed for malignant intracerebral artery stroke. The purpose of this study is to investigate the characteristics and prognostic factors of patients undergoing DC after acute ischemic stroke.

Method From 2013 to 2018, we analyzed the prospective gathered data of 58 consecutive patients treated with DC after acute ischemic stroke in a single center. Also, we analyzed the prospective gathered data of 160 consecutive patients treated with mechanical thrombectomy using stent-retrievers for acute ischemic stroke in a single center. Demographic, clinical, laboratory, and radiologic features of each patient were investigated. We compared the characteristics of patients who underwent DC with those of patients who received medical treatment.

Results Of the total 3884 acute ischemic stroke patients, 58 patients(1.49\%) were underwent DC. Of 289 patients who underwent mechanical thrombectomy due to acute ischemic stroke, DC was performed in 32 patients(11.07\%). Of the patients who underwent mechanical thrombectomy due to acute ischemic stroke, post thrombectomy complications $(100 \%$ vs $22.81 \%, \mathrm{p}=0.000)$, preprocedural TICI grade $(p=0.001)$ were significantly higher in patients who underwent DC compared to patients who did not receive craniectomy. 30 days mRS scores(5.13 vs 3.37, p=0.000), 90 days mRS scores(5.06 vs $3.17, \mathrm{p}=0.000)$, ICU hospital days $(21.53$ vs $8.65, \mathrm{p}=0.000)$ and hospital mortality $(46.88 \%$ vs $12.5 \%$, $\mathrm{p}=0.000$ ) were significantly higher in the craniectomy patients.

Conclusions Patients who underwent DC had more post procedural complications, and the functional outcomes were worse after DC in acute ischemic stroke. However, since DC is the last treatment option for patients with severe cerebral infarction who are not responding to medical treatment, it is advisable to consider active management while managing the underlying disease and periprocedural complications.

Disclosures K. Jang: None. B. Cho: None. B. Moon: None. K. Kim: None. D. Jang: None.

\section{Late-Breaking Oral Abstracts}

\section{THE RIVER TRIAL: A TRIAL OF VENOUS SINUS STENTING WITH THE RIVER STENT IN IDIOPATHIC INTRACRANIAL HYPERTENSION (IIH)}

A Patsalides*. Weill Cornell Medical College, New York, NY

\subsection{6/neurintsurg-2019-SNIS.224}

Introduction Venous sinus Stenting (VSS) is an emerging treatment for patients with IIH. However, VSS is currently performed with stents designed for stenting atherosclerotic arterial stenosis. The River ${ }^{\mathrm{TM}}$ stent has been specifically designed for treating the stenosis of the transverse-sigmoid sinuses and has the following characteristics: easy navigability through the tortuosity of the sigmoid sinus; $7 \mathrm{~cm}$ long length to cover the venous sinuses from the torcula to the mid sigmoid segment; two zones of radial force to address the intrinsic stenosis at the sigmoid transverse junction (stiffer zone) and extrinsic stenosis of the mid transverse sinus (softer zone); and large open cells to adapt to the triangular shape of the venous sinus without occluding cortical veins.

Methods The River trial is a single arm pivotal trial designed for HDE approval of the River ${ }^{\mathrm{TM}}$ stent (Serenity Medical Inc, Redwood City, CA). The trial will enroll 39 subjects in 7 clinical sites. Subjects must have proven $\mathrm{IIH}$ and $>50 \%$ stenosis of the sigmoid-transverse sinus with pressure gradient $\geq 8 \mathrm{~mm}$ $\mathrm{Hg}$. Subjects can be enrolled on visual criteria: visual field deficit from $-6 \mathrm{db}$ to $-30 \mathrm{db}$ resistant to medical therapy $>2$ weeks; or on headaches criteria: severe headaches defined by Headache Impact Test (HIT-6) $>59$ resistant to medical therapy $>4$ weeks. The patients need to be on dual antiplatelet therapy (aspirin and clopidogrel) for 3 months and aspirin monotherapy for 9 more months.

The primary safety endpoint is the rate of major adverse event (any neurological complication plus need for any surgical procedure for $\mathrm{IIH}$ ) at 12 months. The primary efficacy endpoint is a composite at 12 months of clinical improvement and absence of significant venous stenosis. Secondary endpoints at 12 months include opening pressure on lumbar puncture, individual ophthalmology endpoints, change in pulsatile tinnitus, change in medications, change in quality of life scores.

Results Enrollment started in September 2018. Four sites are active at the time of submission of this abstract and three other sites are in the process of certification. Eleven subjects have been enrolled. In all 11 cases the catheterization with the River $^{\mathrm{TM}}$ stent was extremely easy and the stent was deployed as expected. In two patients with severe stenosis from arachnoid granulation, post-stent angioplasty was performed. There was resolution of the trans-stenotic gradient in all cases. There was no stent-thrombosis. There was no impairment of flow in the cortical veins draining into the stented segment. There were no major neurological adverse events or device related complications.

None of the enrolled patients reached the 12-month follow-up time point and therefore we cannot report on primary 